\title{
LATINO SPOTLIGHT
}

\section{Room to Grow}

Latinos 50-plus: Early adopters, tech

spenders, and interested learners

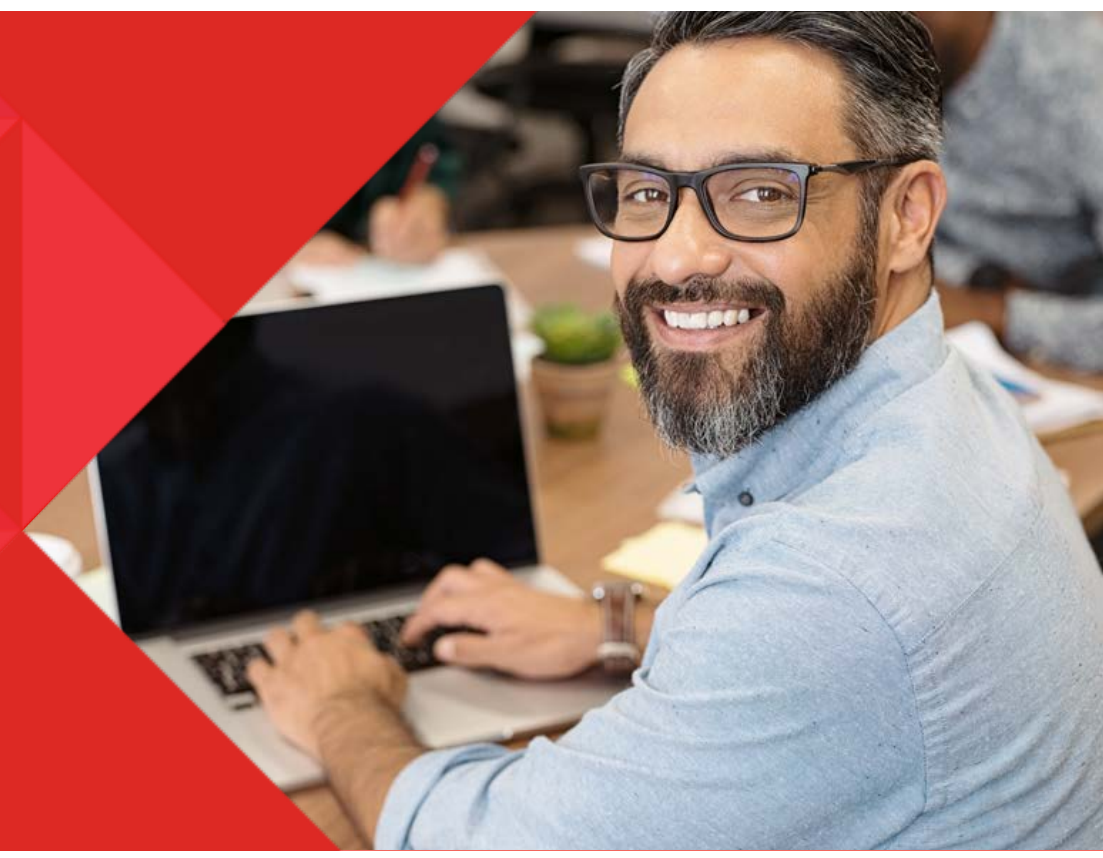

Latinos 50-plus are rather dichotomous. On the one hand, they are more likely than their peers to purchase new technology and, on the other, they did not increase technology use during COVID-19 quarantines. This population relies on technology to stay in touch with family and friends and is eager to learn how their daily lives can be improved through incorporating more technology, like home assistants and smart home devices.

\section{Latino 50-plus use smartphones to stay connected to loved ones}

With nearly universal daily use (99\%), smartphones are used by a majority of Latinos 50-plus to text (95\%), get directions or traffic information $(81 \%)$, visit websites or surf the internet $(81 \%)$, get news and other information (78\%), download or purchase an app (74\%), and access a social networking site (69\%). Be it using a smartphone or a tablet, the most popular apps among this cohort involve email $(80 \%)$, browsing the internet $(68 \%)$, photos $(73 \%)$, social media $(65 \%)$, weather $(60 \%)$, and shopping (54\%).

Four in five (79\%) Hispanic/Latinos use technology to stay connected with friends and family. On a daily basis, a majority use text messaging $(75 \%)$ to stay in touch, and on a weekly basis, while texting still reigns (91\%), a majority also email $(68 \%)$ or use social media (64\%), most often Facebook (87\%).
A new study examines how Latinos 50-plus use technology 


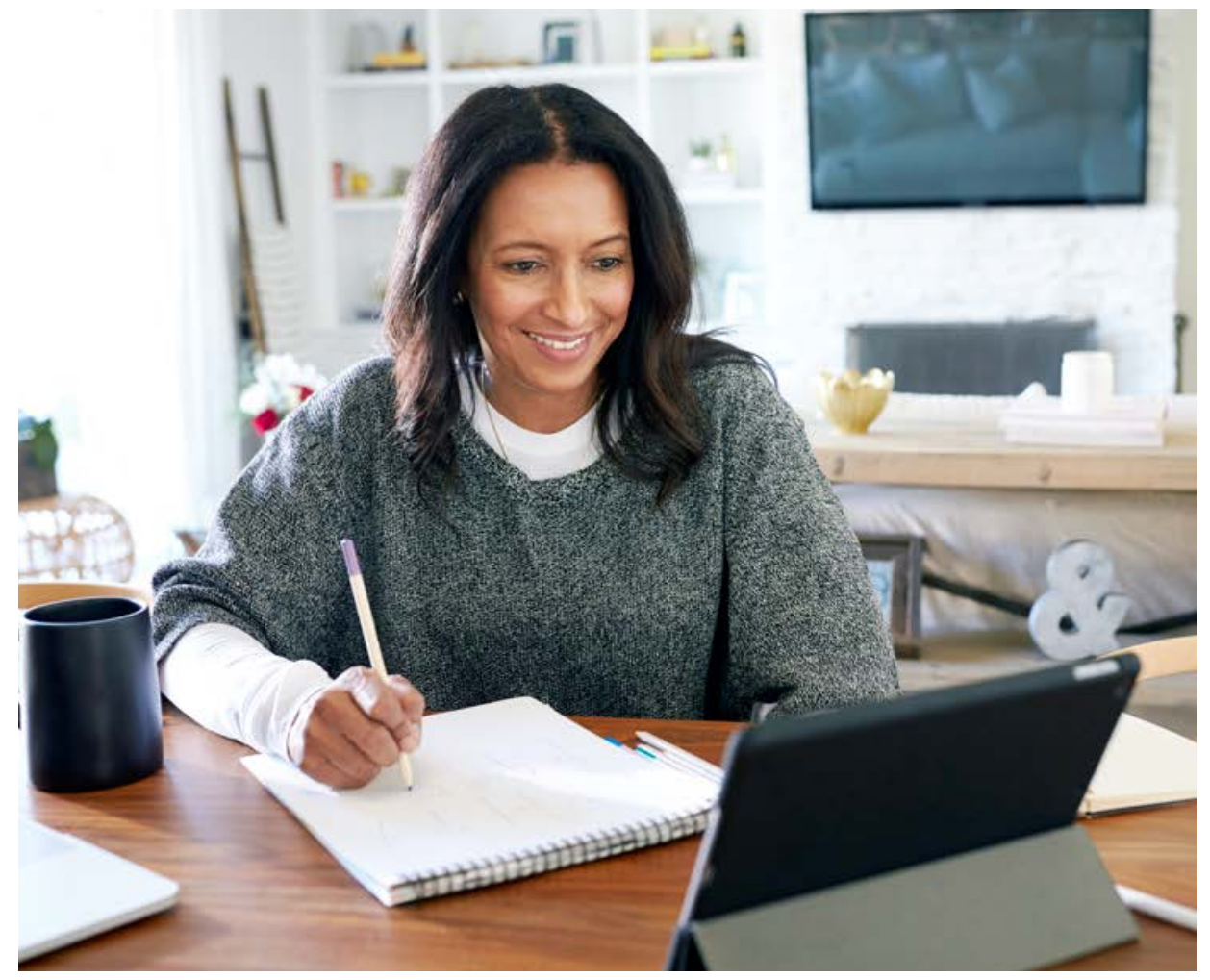

\section{Most are comfortable with technology, but are open to learning more about it}

Many older Hispanics were video chats power users before pandemic stay-in-place orders forced many to turn to technology to stay connected with friends and family. In 2019, $69 \%$ of older Latinos used video chat, compared to $45 \%$ of the total 50 -plus audience. In $2020,75 \%$ of older Latinos use video chat (compared to $70 \%$ of the 50 -plus). Older Latinos video chat with high rates of monthly (66\%) and weekly use (46\%). One in seven video chat daily (15\%). For many, video chat has long been a way to stay connected with loved ones near and far.

While three in five $(63 \%)$ report overall feeling confident in their use of technology, nearly as many $(57 \%)$ wish they had a better grasp of technology. Examining this distinction sheds light on areas of opportunity for growth. For example, roughly two-thirds of all Hispanic/ Latinos 50-plus feel comfortable using smartphones (67\%) and computers $(63 \%)$, nearly the same amount as those who own one $(73 \%$ and $71 \%$, respectively). However, the comfort-level disparity grows between owners of home assistants (54\%) and tablets (74\%) and Hispanic adults 50 -plus overall (29\% and $53 \%$, respectively), as those who are not necessarily owners are much less comfortable with such technologies.
Percent who use tech devices daily

Among Hispanics 50-plus

$99 \%$

Smartphone

$80 \%$

Smart Home Technology

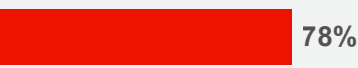

Wearable Device

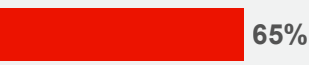

Home Assistant

$56 \%$

Laptop Computer

$56 \%$

Desktop Computer

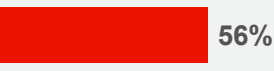

Tablet

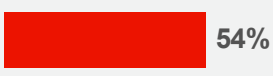

Regular Cellphone $39 \%$

GPS Tracker

$16 \%$

E-Reader

$3 \%$

Virtual Reality 
To learn about new technologies, about one-quarter $(27 \%)$ want to have friends and family show them and one in five $(21 \%)$ prefer to teach themselves using online tutorials and videos. Hispanic/Latinos 50-plus would most benefit from learning how to use a home assistant, like Google's Home and Amazon's Echo or Dot, or how to manage or monitor smart home technology, like Ring doorbells and Nest thermostats - something one-quarter say they would use daily if they knew how (29\% and $25 \%$, respectively).

\section{Hispanic/Latinos 50-plus Comfortable with Technology}

Owners $\square$ All

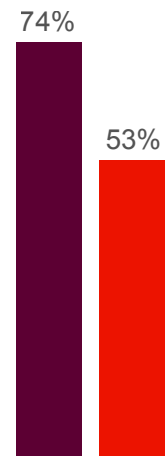

Tablets

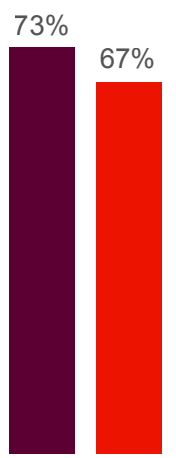

Smartphone

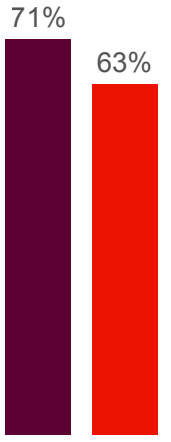

Computers

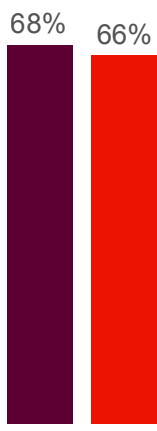

Internet

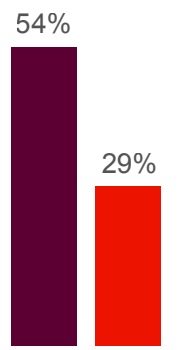

Home assistants

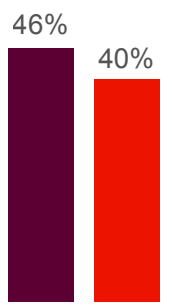

Video chat

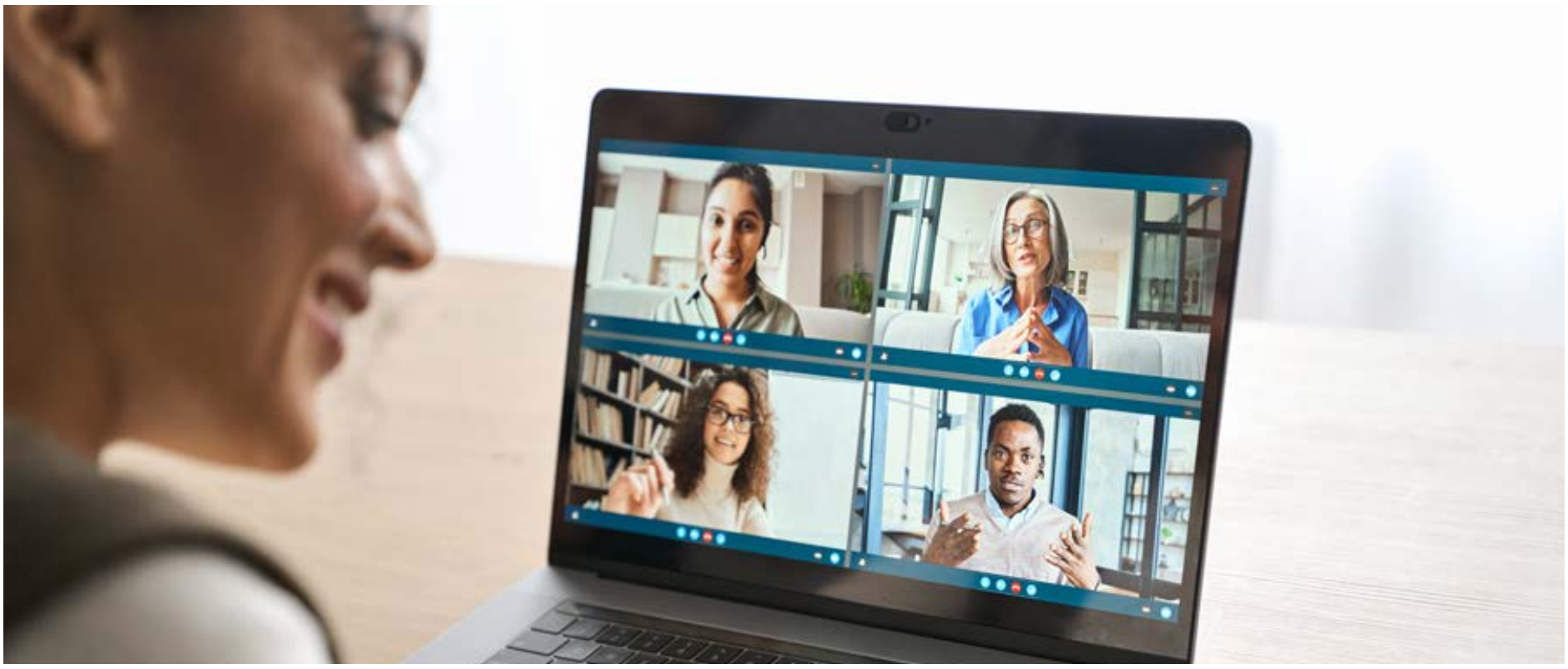

\section{Technology use during COVID-19 maintained strong for many}

During COVID-19, when the world was in varying stages of lockdowns and stay-at-home orders, it would be reasonable to assume many turned to technology to bridge the social gap, but many Hispanic/Latinos 50-plus were already using technology to stay connected so there were few significant increases. While the technology use for many did not increase, the lone exception is attending virtual live events $(24 \%$ increased, compared to $18 \%$ stayed the same). 
Three in five (61\%) Hispanic/Latinos 50-plus are familiar with live virtual events and almost one-third (30\%) have participated in one since the start of COVID-19. When selecting what events to attend, many factors play a near-equal role, from cost $(21 \%)$ to the day and time $(24 \%)$, the type of the event $(20 \%)$ to the hosting organization or person (17\%). Among the seven in ten $(70 \%)$ who have not attended an online event, most simply have no interest (44\%) while others do not want to pay for them (26\%), don't feel like doing anything right now $(20 \%)$, or don't know of any $(18 \%)$.

\section{COVID-19's Impact on Technology Use}

The same amount $\quad$ Increased amount

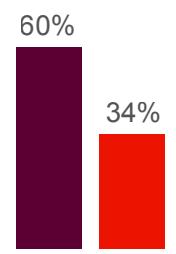

Phone call

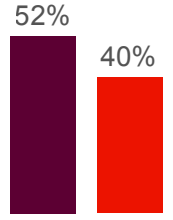

Text

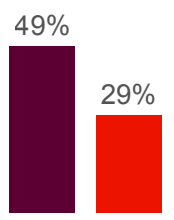

Email

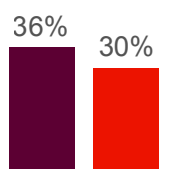

Video chat

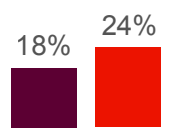

Live virtual events

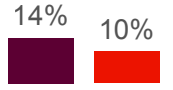

Virtual/remote volunteering

\section{More purchasing power than most, but costs are a concern}

Eighty-six percent of older Latinos own a smartphone. The top three purchases were smartphones, smart tv's, and Bluetooth headsets/headphones. In fact, Latino 50-plus adults were more likely than their peers to purchase a new smartphone (42\%, compared to $36 \%$ ), a smart TV (31\%, compared to $24 \%$ ), a Bluetooth headset $(28 \%$, compared to $23 \%$ ), and a tablet $(22 \%$, compared to $17 \%)$. Dollar for dollar spending by Hispanic/Latino adults 50-plus is up from $\$ 538$ in 2019 and on par with all adults 50-plus (\$1,163, compared to $\$ 1,148)$.

$67 \%$ Two-thirds of Hispanic/Latinos 50 plus own Smart TVs

\section{Technology Ownership Among 50-plus Hispanics}

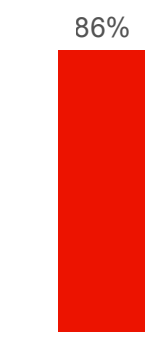

Smartphone

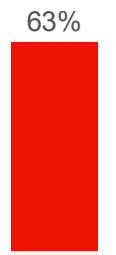

Laptop computer

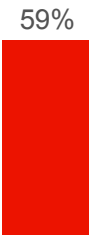

Tablet

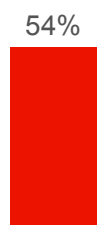

Desktop computer
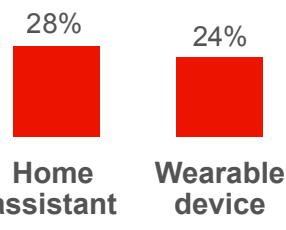

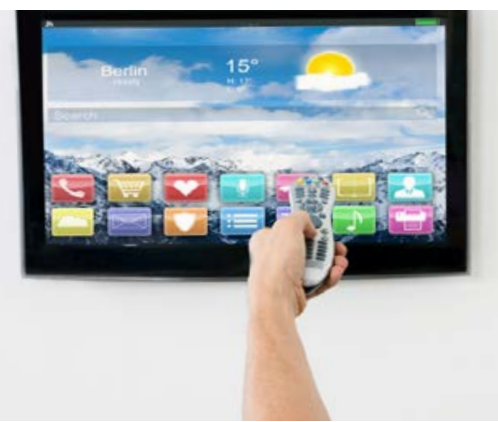

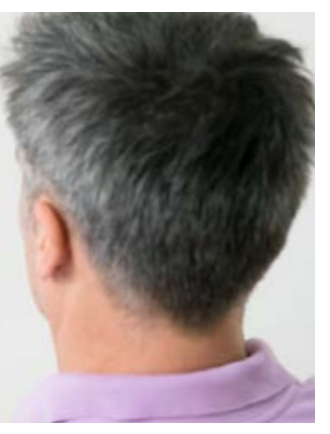




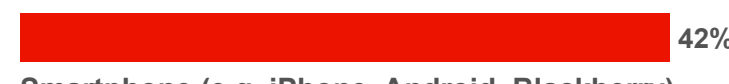

Smartphone (e.g. iPhone, Android, Blackberry)

$31 \%$

Smart TV

$28 \%$

Bluetooth headset/ear buds (e.g. AirPods)

\section{$15 \%$}

Laptop computer or Netbook $22 \%$

Tablet (e.g. iPad)

$11 \%$

Wearable device (e.g. Fitbit, Garmin, Apple Watch)

$13 \%$

Home assistant

$13 \%$

Smart Home Technology/security (e.g. a smart thermostat, Nest, Ring, SimpliSafe)

\section{$8 \%$ \\ Desktop computer}

$6 \%$

Home audio equipment (e.g. Sonos)

$6 \%$

Digital camera

$3 \%$

E-reader (e.g. Kindle, Nook)

$7 \%$

Gaming system

$3 \%$

GPS Tracker specifically designed to monitor and share location (e.g. a necklace, bracelet or watch)

$2 \%$

Home health and safety device (e.g. emergency alert systems, medication manager)

$2 \%$

Virtual reality (VR) device

While many made new technology purchases, costs associated with using technology are a concern for many. On average older Hispanic adults spend $\$ 273$ a month-19\% of their budget (similar to the general 50-plus market average of $\$ 269-16 \%$ ) on tech expenses such as internet, cellphone, cable, and streaming services. The average estimated monthly costs are internet, $\$ 67$; cellphone, $\$ 109$; cable, $\$ 77$; and streaming services, \$20).

Rising technology costs are pushing some older Hispanic/Latino adults away from cable to streaming platforms while some still maintain traditional viewing habits. There are similar rates of older Hispanics who have completely cut the cord and only watch streaming services (15\%) as there are those who only watch network TV $(18 \%)$ and do not stream at all. The rest are in the middle. Netflix subscriptions are up from $62 \%$ to $73 \%$.

\section{Top Streaming Platforms}
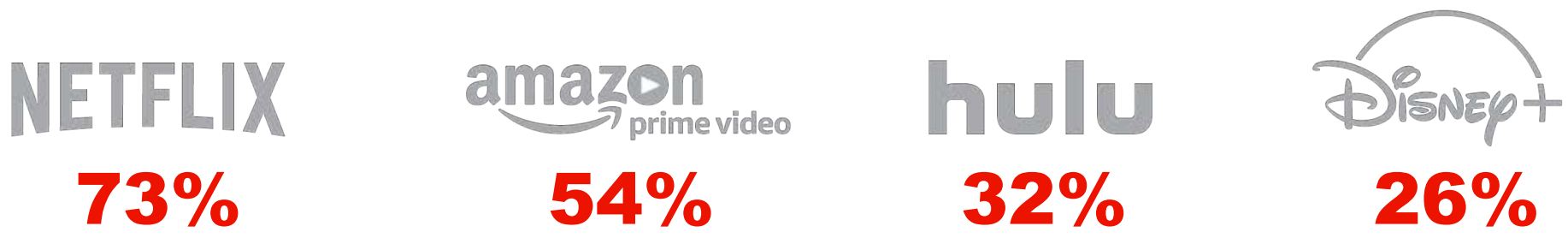


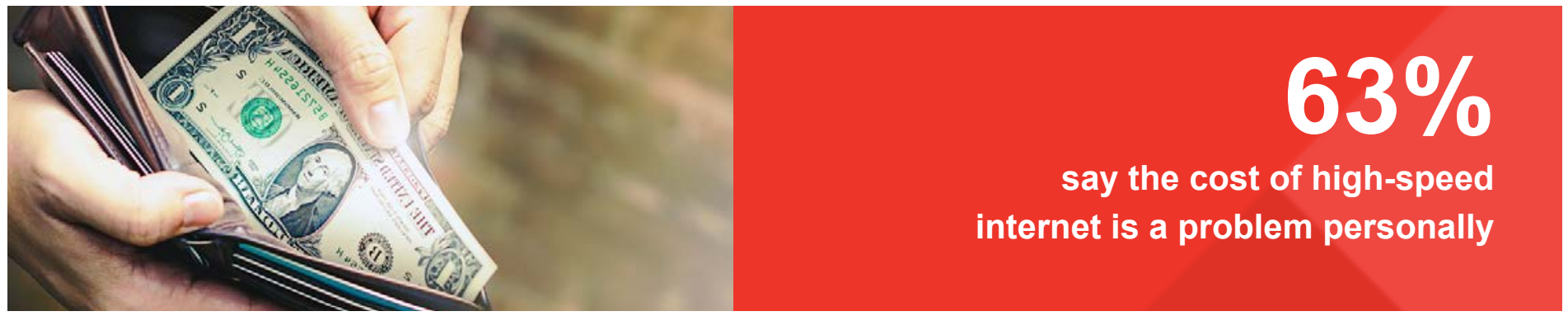

\section{Privacy concerns could tamper technology adoption and use}

While many older Latinos have embraced technology to manage their daily lives and connect with others, privacy concerns about sharing of personal information could inhibit future technology adoption. Privacy concerns $(19 \%)$ rather than cost $(11 \%)$ is the top-ranked barrier holding older Hispanics say back from adopting new tech. This differs from their peers where cost is the number one barrier. When considering first, second, or third ranked concerns, privacy ties with cost as overall barrier to adopting technology. (privacy-36\%, cost-36\%, knowledge-34\%, user experience-23\%, lack of interest-22\%).

Unfortunately, while many are concerned about privacy there is little understanding when it comes to privacy policies. Most were incorrect in believing when a website has a privacy policy, it means the site will not share their information with other websites and companies without their permission. (33\% answered incorrectly, 33\% answered unsure, only $34 \%$ answered correctly-FALSE). Older Hispanics need clear communication addressing their privacy concerns as well as education about what online privacy means.

\section{Implications}

Instead of waiting for Hispanic/Latinos 50-plus to purchase various technology devices and become comfortable with them, it is possible increased levels of comfort may drive increased ownership rates. While many are comfortable with smartphones, ownership of other types of technology lag. Therefore, tablet, wearable, and home assistant companies should find creative ways to help this population become comfortable with these technologies. Fortunately, many like learning about technology through online tutorials and videos and, at the rate they frequent Facebook and other social medial platforms, this may be a perfect place to strategically post bi-lingual, short, introductory videos to familiarize Hispanic/Latinos with these and other technologies. Hispanic/Latinos 50-plus are buying new technological devices, from smart TVs to Bluetooth headsets, even if they have privacy concerns. Tech companies would be wise to provide clear and transparent communications to this demographic through advertising as they are eager and willing to invest in various technologies and use them frequently once owned.

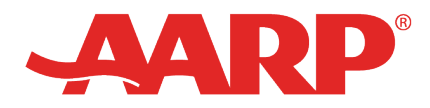

For more information on the survey and methodology please contact:

Brittne Kakulla, bkakulla@aarp.org

DOI: $\underline{\text { https://doi.org/10.26419/res.00420.013 }}$

2021 Tech Trends and the 50-Plus: Top 10 Biggest Trends. Washington, DC:

AARP Research, April 2021. DOI: https://doi.org/10.26419/res.00420.001 